\title{
In vitro antifungal activity of essential oils from local plants against fluconazole-resistant oral Candida albicans isolates
}

\author{
Sazan Moffaq Abdulaziz * Ibrahim Abdulrahman Shaswary ** Aso Ako Muhammad *
}

\section{Abstract}

Background and objective: Candida albicans is an oral commensal flora that causes opportunistic local and systemic infections in immunocompromied individuals. Fluconazole is frequently used for treating patients with active infections or preventing recurrent infections. The emergence of resistant strains encouraged scientists to search for compounds that have antifungal property and can overcome the usual microbial resistant mechanisms to antimicrobial agents. Essential oils from natural plants have received great interest due to the antimicrobial property of their multiple constituents. The aim of this study was to investigate the antifungal activity of Rosemary and Thyme essential oils against fluconazole resistant oral Candida albicans isolates.

Methods: Essential oil from areal parts of Rosemarium officinalis and Thymus vulgaris were obtained by hydrodistillation. Disk diffusion and microbroth dilution methods were followed to test the sensitivity of eight fluconazole resistant oral Candida albicans isolates and one ATCC strain to the extracted Essential oils. Minimum inhibitory concentration and minimum fungicidal concentration were used to evaluate the antifungal potential of the tested essential oils.

Results: The results of disk diffusion method using concentrated Essential oils showed marked inhibition of growth around the prepared discs from both Essential oils. However, the recoded diameters was higher in Thyme Essential oil (Mean \pm SD: $42.4 \pm 6.5$ ) when compared with Rosemary essential oil (Mean \pm SD: $11.8 \pm 2.8$ ). Serial two fold dilutions of the tested essential oils showed that both essential oils attain their antifungal activities even at very low concentrations. The highest MIC and MFC of Rosemary EO were $3.125 \%$ and $6.25 \%$, respectively. Thyme essential oil showed very low MIC and MFC $(<1.56 \%)$.

Conclusion: Thyme and Rosemary essential oils were ideal natural compounds against drug resistant Candida albicans strains.

Keywords: C. albicans, Fluconazole, Essential oil, Rosemarium officinalis, Thymus vulgaris.

\section{Introduction}

Species of the genus Candida are the most common fungal pathogens responsible for the majority of human infections ranging from localized superficial to systemic candidiasis. ${ }^{1,2}$ In the oral cavity, among various Candida species, Candida albicans (C. albicans) comprises the most prevalent one in health and disease. ${ }^{3,4}$ It is an opportunistic pathogen as it usually causes infection in individuals with low or suppressed immunity. 5,6 Fluconazole is one of the widely used azole drugs in the clinical settings to treat infections caused by $\mathrm{C}$. albicans or in the prophylaxis to prevent recurrent candidiasis in immunocompromised patients. ${ }^{7}$ In term of increased antimicrobial resistance among pathogenic microorganisms due to indiscriminate use of antimicrobial drugs, the focus has been directed toward medicinal plants for the treatment and prevention of various infectious diseases. ${ }^{8}$ In a World health organization (WHO) survey, it was reported that traditional medicine using plant extracts are used by

* Department of Basic Science, College of Dentistry, Hawler Medical University, Erbil, Iraq

** Scientific Research Center, University of Zakho, Duhok, Iraq 
the majority of world's populations as a primary treatment of many diseases. ${ }^{9}$ Relatively, low side effects, availability and enrichment in a variety of biologically active compounds against many microorganisms are the main reasons behind their increased acceptance. ${ }^{10}$ Essential oils (EOs) are natural complex compounds extracted from different parts of aromatic plants (leaves, flowers, seeds, roots, etc..) with strong and characteristic odor. They are used in different aspects of life especially in pharmacology, medicine, agriculture, and food production. Chemically, each EO is a mixture of nearly $20-60$ different constituents, two or three are major components found in high concentrations in the extracted oil. Phenolic compounds, terpenoids and alkaloids are examples of the major constituents. These in turn include many active compounds. For example, phenolics include alcohols, aldehydes and acids that have great impact on living cells. ${ }^{11,12}$ They seem to have no specific targets, ${ }^{13}$ inducing ultrastructural alterations of microbial cell compartments including cell wall, cell membrane, inner and outer membrane of mitochondria, cytoplasm and also nucleus. Due to their lipophilic action, EOs can easily pass through membranes causing leakage of cell materials and finally death. ${ }^{14,15}$ Rosemary and thyme are examples of aromatic plants enriched in these important bioactive compounds known for their antibacterial, antifungal, antiviral, antioxidant and anti-inflammatory activities. ${ }^{16}$ Since components of the EOs differ from place to another depending on the soil, climate and according to the methods of extraction. ${ }^{17}$ The aim of this study was to investigate the antifungal activity of laboratory made EOs from local Rosemary and Thyme plants against C. albicans strains that showed high resistance to fluconazole isolated from the oral cavity, in an attempt to contribute to the use of these oils as an alternative treatment for oral candidiasis.

\section{Methods}

\subsection{Place of study}

The practical work of this research was shared between College of Dentistry, Hawler Medical University (HMU), Erbil, Iraq and Scientific Research Center, University of Zakho (UOZ), Duhok, Iraq.

\subsection{Plant material}

The areal parts of Thymus vulgaris and Rosemarium officinalis were collected in spring, May 2013, in Erbil, Iraq. The collected plant samples were sent to the Scientific Research Center of University of Zakho, Duhok, Iraq for botanical study and isolation of the EOs.

\subsection{Essential oil isolation}

EOs from both plants were extracted by hydrodistillation of the collected plant samples for $3 \mathrm{~h}$ using a Clevenger-type apparatus. $^{1}$ The yield was $1 \%$ and $0.6 \%$ for Rosemarium officinalis and Thymus vulgaris, respectively. Purity of the extracted oils was checked and estimated to be $>99 \%$. Dimethylsulfoxide (DMSO) was added to the extracted EOs in concentrations ranged from 5 to $10 \%(\mathrm{v} / \mathrm{v})$ to enhance their solubility and diffusion.

\subsection{Test organism}

Eight clinical isolates of $\mathrm{C}$. albicans that showed highly resistant potential to fluconazole $(>128 \mu \mathrm{g} / \mathrm{ml})$ in our previous study (4) and a reference strain (ATCC 10231) were included. All these isolates were maintained on sabouraud dextrose agar slants at $4^{\circ} \mathrm{C}$ and subcultured monthly throughout the study period.

\subsection{Antifungal activity of the EOs}

\subsubsection{Disc diffusion method}

Simple disc diffusion method was applied for screening the antifungal activity of Rosemary and Thyme EOs against C. albicans isolates. ${ }^{18}$ To prepare EO containing discs, ten microliters of each EO was added to sterile $6 \mathrm{~mm}$ filter paper discs (WHATMAN no.3) and let to dry. Discs that served as positive and negative controls were prepared by the addition of $10 \mu \mathrm{l} / \mathrm{disc}$ sterile normal saline $(0.9 \%)$ and chlorhexidine solution $(0.12 \%)$, respectively. As a vehicle control, another group of discs were prepared from aqueous DMSO using the same method described above. To perform the disc 
diffusion method, the inoculum from each C. albicans isolate was prepared from a fresh 18 hour growth on SDA plates through emulsifying 2-3 discrete colonies in $5 \mathrm{ml}$ of sterile normal saline $(0.9 \%)$. The turbidity was adjusted to a 0.5 McFarland, using spectrophotometer (BIO-TEK Instrument, Milan, Italy) at 450nm wavelength. Then, a sterile swab soaked in the prepared inoculum and spread on the entire surface of a SDA plates in different directions to ensure the uniform spread of the inoculum. The inoculated plates were left to dry at room temperature. Later, the previously prepared discs were placed on the surface of the inoculated medium and incubated for $24 \mathrm{~h}$ at $37^{\circ} \mathrm{C}$, after which the diameter of inhibition zones were recorded.

\subsubsection{Microbroth dilution method}

Microbroth dilution method mentioned by Singh et $\mathrm{al}^{19}$ was used with some modifications to determine Minimum inhibitory concentration (MIC) and minimum fungicidal concentration (MFC) of the tested EOs. Six dilutions from each EO in sabouraud dextrose broth $(50 \%, 25 \%$, $12.5 \%, 6.25 \%, 3.125 \%$ and $1.56 \%$ ) were used. This test was performed in sterile 96-well microtiter plates (Deltalab, Spain). Row A has received $50 \mu$ l of the tested EO in DMSO solution, while the remaining wells, B to $G$ received $50 \mu l$ of sabouraud dextrose broth (SDB). Serial two-fold dilutions were prepared vertically in the plate. Excess dilution $(50 \mu \mathrm{l})$ was discarded from row $\mathrm{G}$. Then $30 \mu \mathrm{l}$ of the inoculum containing $10^{7}$ cells $/ \mathrm{ml}$ was added to each well. Two other wells were served as positive control (no extract was added) and negative controls (chlorhexidine was added instead of the EOs). The plates were incubated at $37^{\circ} \mathrm{C}$ for $24 \mathrm{~h}$. In order to decide on and confirm the primary decision on the MIC and MFC of the tested EOs, $10 \mu \mathrm{l}$ of broth from the first well that showed no clear growth and another two successive clear wells onto SDA plates. Then, the inoculated plates were incubated for $24 \mathrm{~h}$ at $37^{\circ} \mathrm{C}$. The MIC was defined as the lowest concentration of the EO that inhibited visible growth of C. albicans isolates within in the well. On the other hand, the MFC was defined as the lowest concentration of the EOs that completely inhibited growth of Candida isolates on SDA plates. Each test was repeated twice and the average inhibitory and cidal concentrations were recorded.

\subsubsection{Statistical analysis}

The statistical package for the social sciences (version 20.0) was used to analyze data and Excel 2010 for figures. t-test was used to find out the significant difference between the means of the recoded diameter of growth inhibition zones of the tested EOs. A $P$ value equal or less than 0.05 was considered statistically significant.

\section{Results}

The results of disc diffusion method for testing the antifungal activity of both Rosemary and Thyme EOs are summarized in Figure 1. It was found that both EOs are active against the selected fluconazole resistant $C$. albicans isolates. Thyme EO showed an obviously higher inhibitory activity (Mean \pm SD: $42.4 \pm 6.5$ ) than Rosemary EO (Mean \pm SD: $11.8 \pm$ 2.8). However, statistically this difference was not significant $(P=0.153)$. Zones of growth inhibition were not seen around discs impregnated with DMSO and normal saline $(0.9 \%)$, indicating no antifungal activity of these solutions.

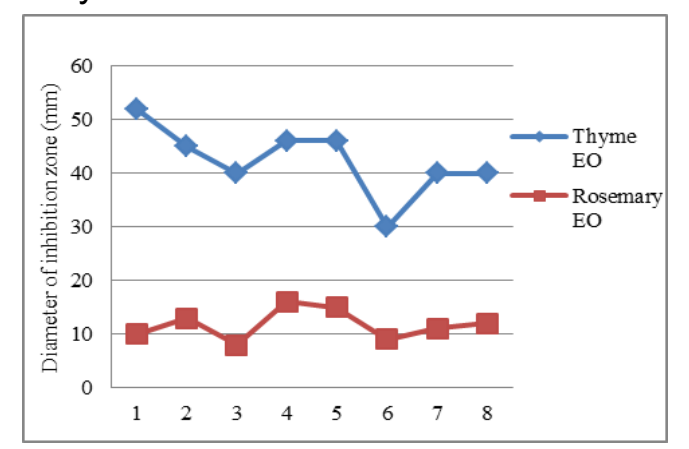

Figure 1: Antifungal activity of the tested EOs against eight fluconazole-resistant C. albicans isolates using disc diffusion method. 
The same isolates were subjected to two fold dilutions of the tested EOs to reach their MIC and MFC. It was found that both EOs had potent antifungal activities even in very low concentrations (Table 1). The highest MIC and MFC of Rosemary EO were $3.125 \%$ and $6.25 \%$, respectively. Thyme EO showed very low MIC and MFC (<1.56\%). Microscopical examination of prepared wet smears was done to assess the effect of the tested EOs on the growth rate and morpholology of treated C. albicans cells in comparision to untreated (control) cells (Figure 2). Round to oval shape cells showing active growth were characteristic of Candida cells in the control group (A). Reduced growth and change in normal cell arrangement were seen in EO treated cells at MIC (B). Greatly reduced cell size, number and cell deformity were observed at MFC (C) indicating the fungicidal activity.

Table 1: MICs and MFCs of the tested EOs against eight fluconazole-resistant C. albicans isolates.

\begin{tabular}{llll}
\hline C. albicans isolates & $\begin{array}{l}\text { Rosemary EO } \\
\text { MIC (\%) }\end{array}$ & MFC (\%) & $\begin{array}{l}\text { Thyme EO } \\
\text { MIC \& MFC (\%) }\end{array}$ \\
\hline 1 & 1.56 & 3.125 & $<1.56$ \\
2 & 3.125 & 6.25 & $<1.56$ \\
3 & 1.56 & 3.125 & $<1.56$ \\
4 & $<1.56$ & $<1.56$ & $<1.56$ \\
5 & $<1.56$ & $<1.56$ & $<1.56$ \\
6 & $<1.56$ & $<1.56$ & $<1.56$ \\
7 & $<1.56$ & $<1.56$ & $<1.56$ \\
8 & 3.125 & 6.25 & $<1.56$ \\
ATCC 10231 & $<1.56$ & $<1.56$ & $<1.56$ \\
\hline
\end{tabular}

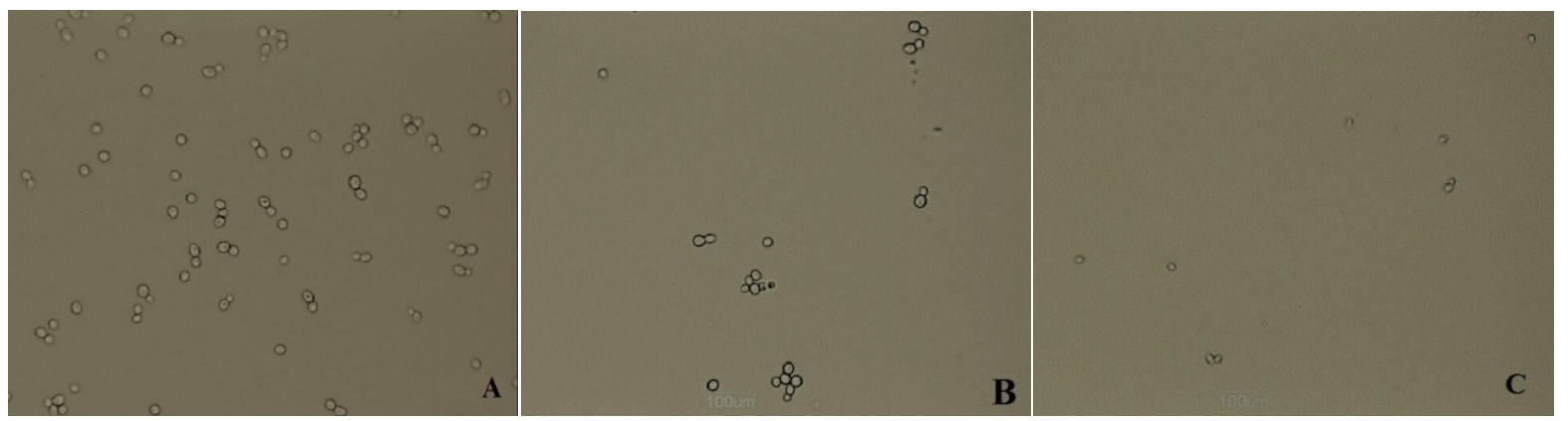

Figure 2: Microscopical examination of treated and untreated (control) C. albicans cells with Rosmary EO following $24 \mathrm{~h}$ incubation at $37^{\circ} \mathrm{C}$. (A) represents untreated cells, (B) and (C) treated cells at MIC and MFC, respectively. 


\section{Discussion}

The main goal behind the vast variety of nowadays studies interested in the use of extracts from natural plants in the medical field is to introduce a scientifically and popularly acceptable material that may compensate the use of antimicrobial drugs in treating infectious diseases, especially those caused by multidrug resistant strains that might be difficult to manage even with the use of multidrug therapy. Reports about opportunistic infections caused by C. albicans are increasing all over the world, and the number of reports on strains resistant against commonly used antifungal drugs is also in progress. ${ }^{20}$ This study was conducted to assess the antifungal activity of the laboratory extracted Rosemary and Thyme EOs against fluconazole-resistant C. albicans strains isolated from the oral cavity of healthy and diseased individuals from our population. ${ }^{4}$ In the present study, it was found that both EOs exhibited significant antifungal activity against $\mathrm{C}$. albicans. In spite of the higher inhibitory activity of Thyme EO using disk diffusion method (Mean \pm SD: $42.4 \pm 6.5$ ) in comparison to the recorded data regarding Rosemary EO (Mean \pm SD: $11.8 \pm 2.8$ ), the statistical analysis showed no significant difference between the two means. This result could be attributed to the low number of the tested C. albicans isolates included in this study. Similarly, Thyme EO showed much higher inhibitory and cidal potentials $(<1.56 \%)$ than Rosemary EO $(3.125 \%$ and $6.25 \%$, respectively) using microbroth dilution method, as it was effective at very low concentrations against all the tested strains. These results are consistent with those observed by previous researches in which both EOs showed good antifungal potentials against different Candida species at very low concentrations. ${ }^{21-23}$ Two possible reasons may explain the greater antifungal activity of Thyme EO than Rosemary EO. It was reported by Chandra et $\mathrm{al}^{25}$ that the expression of fluconazole-resistant gene in C. albicans might be responsible for the increased MIC of the tested chemical compounds against the fluconazole-resistant strains. This finding might be accounted for the increased MIC and MFC of Rosemary EO over Thyme EO observed among the tested $\mathrm{C}$. albicans isolates. Variations in the susceptibility pattern of the tested $\mathrm{C}$. albicans isolates to each $\mathrm{EO}$ are influenced by the permeability of the cell surface to the tested EO. ${ }^{24}$ On the other hand, the antifungal activity of the EOs could be attributed to their complex mixture of biologically active compounds that are greatly differ according to the chemotype of the plant and the place from which the plant was obtained. ${ }^{26}$ Previous chemo-microbiological investigations have identified carvacrol and thymol as the major compounds in Thymus vulgaris $\mathrm{EO},{ }^{27,28}$ and pinene, limonene and piperitone in Rosmarium officinalis with active antifungal properties, all of which are phenolic compounds. ${ }^{29}$ Dependingly, these active compounds have the ability to diffuse into viable cells and interfere with normal cell's metabolic activities. Assessment of the effect of increasing concentration of the tested EOs on active growth and morphology of $\mathrm{C}$. albicans cells showed a decrease in cell number at the MIC in comparison to the control cells. This result could be attributed to the fact that certain components in EOs react with enzymes responsible for cell wall synthesis thereby decreasing the rate of cell division. ${ }^{30}$ Increasing in the EO concentration above the MIC shifted the growth curve more toward the death phase (MFC), as the bioactive properties of different constituents in EOs results in multiple damages and functional impairments of fungal cell compartments resulting in altered cell morphology, growth failure and even difficulty to survive in such environment. ${ }^{14,15}$

\section{Conclusion}

Both Rosemary and Thyme EOs could be regarded as active natural bioactive compounds against fluconazole-resistant 
oral C. albicans isolates. Further investigations are required to prove their in vivo activity and safety. It is necessary to chemically analyze the tested EOs to exactly determine their composition and to assess the antimicrobial property of their major constituents.

\section{Conflicts of interest}

The authors report no conflicts of interest.

\section{References}

1. Pinto E, Vale-Silva L, Cavaleiro C, Salgueiro L. Antifungal activity of the clove essential oil from Syzygium aromaticum on Candida, Aspargillus and dermatophyte species. J Med Microbio 2009; 58:1454-62.

2. Sahand IH, Maza JL, Eraso E, Montejo M, Moragues MD, Aguirre JM, et al. Evaluation of CHROM-Pal medium for the isolation and direct identification of Candida dublineinsis in primary cultures from the oral cavity. J Med Microbiol 2009; 58:1437-42.

3. $\mathrm{Xu} \mathrm{J}$, Mitchell TG. Geographical differences in human oral yeast flora. CID 2002; 36:221-4.

4. Abdulaziz SM and Muhammad AA. Oral Candida in $\beta$-thalassemia major and healthy population and their fluconazole susceptibility pattern. IJDSR 2014; 2(2):27-31.

5. Abu-Elteen KH, Hamad MA, Salah SA. Prevalence of oral Candida infections in diabetic patients. Bahrain Medical Bulletin 2006; 28(1):1-8.

6. Alberth M, Majoros L, Kovalecs G, Borbas E, Szeged I, Marton IJ, et al. Significance of oral candidiasis infections in children with cancer. Path Oncol Res 2006; 12(4):237-41.

7. Samaranayake L. Essential microbiology for dentistry. $4^{\text {th }}$ ed. Edinburgh: Churchill LivingstoneElsevier; 2012. p. 188.

8. Rapp RP. Changing strategies for the management of invasive fungal infections. Pharmacotherapy 2004; 24:4S-28S.

9. National policy on traditional medicine and regulation of herbal medicines (online). WHO, 2005. (Accessed 2012 Aug 31, at http://apps.who.int/medicinedocs/pdf/s7916e/ s7916e.pdf.)

10. Pirbalouti AG, Bahmani M, Avijgan $M$. Anti-Candida activity of some of the Iranian medicinal plants. eJBio 2009; 5(4):85-8.

11. Silva NCC, Fernandes Junior A. Biological properties of medicinal plants: a review of their antimicrobial activity. J Venom Anim Toxins incl Trop Dis 2010; 16:402-13.

12. Angioni A, Barra A, Coroneo V, Dessi S, Cabras $P$. Chemical composition, seasonal variability, and antifungal activity of Lavandula stoechas L. ssp. stoechas essential oils from stem/leaves and flowers. J Agricult Food Chem 2006; 54:4364-70.
13. Carson CF, Mee BJ, Riley TV. Mechanism of action of Melaleuca alternifolia (tea tree) oil on Staphylococcus aureus determined by time-kill, lysis, leakage and salt tolerance assays and electron microscopy. Antimicrob Agents Chemo 2002; 46:1914-20.

14. Santoro GF, das Gracas CM, Guimaraes LG, Salgado AP, Menna-Barreto RF, Soares MJ. Effect of Oregano (Origanum vulgare L.) and Thyme (Thymus vulgaris L.) essential oils on Trypanosoma cruzi (Protozoa: Kinetoplastida) growth and ultrastructure. Parasitol Research 2007; 100:783-90.

15. Parveen M, Hasan MK, Takahashi J, Murata Y, Kitagawa E, Kodama $O$, et al. Response of Saccharomyces cerevisiae to a monoterpene: evaluation of antifungal potential by DNA microarray analysis. J Antimicrob Chemother 2004; 54:46-55

16. Bakkali F, Averbeck S, Averbeck D, Idaomar M. Biological effects of essential oils - A review. Food Chem Toxicol 2008; 46:446-75.

17. Matsuzaki $\mathrm{Y}$, Tsujisawa $\mathrm{T}$, Nishihara $\mathrm{T}$, Nakamura M, Kakinoki Y. Antifungal activity of chemotype essential oils from rosemary against Candida albicans. OJST 2013; 3:176-82.

18. Cheesbrough M. District laboratory practice in tropical countries. $2^{\text {nd }}$ ed. (part 2). New York: Cambridge University Press; 2006. p. 136-138.

19. Singh R, Singh AK, Soam A, Shahi KS. Antifungal screening of various spice extracts on azole resistant strains of Candida. Current Discovery 2013; 2:46-52.

20. Pina-Vaz C, Rodrigues AG, Pinto E, Costa-deOliveira S, Tavares C, Salgueiro LR, et al. Antifungal activity of Thymus oils and their major compounds. J Eur Acad Dermatol Venereol 2004; 18:73-8.

21. Ahmad I, Beg AZ. Antimicrobial and phytochemical studies on 45 Indian medicinal plants against multi-drug resistant human pathogens. J Ethnopharmacol 2001; 74:113-23.

22. Devkatte AN, Zore GB, Karuppayil SM. Potential of plant oils as inhibitors of Candida albicans growth. FEMS Yeast Res 2005; 5:867-73.

23. Duarte MCT, Figueira GM, Sartoratto A, Rehder VLG, Delarmelina C. Anti-Candida activity of Brazilian medicinal plants. J Ethnopharmacol 2005; 97:305-11.

24. Prabuseenivasan $S$, Jayakumar $M$, Ignacimuthu S. In vitro antibacterial activity of some plant essential oils. BMC Compl Alt Med 2006; 6:39-46.

25. Chandra J, Kuhn DM, Mukherjee P, Hoyer LL, McCormick T, Ghannoum MA. Biofilm formation by the fugal pathogen Candida albicans: development, architecture and drug resistance. J Bacteriol 2001; 183(18):5385-94.

26. Ložiene K, Šakalyte J, Paškevičius A, Venskutonis PR. Anti-Candida activity of Thymus vulgaris (Lamiaceae) essential oils depends on the plant chemotypes. Herba Polonica 2008; 54(4):79-92. 
27. Yanishlieva NV, Marinova MR, Gordon MH, Ravena VG. Antioxidant activity and mechanism of action of thymole and carvacrol in two lipid systems. Food Chem 1999; 64:59-66.

28. Kumar AR, Shukla P, Singh CS, Prasad, Dubey NK. Assessment of Thymus vulgaris L. essential oil as a safe botanical preservative against post harvest fungal infestation of food commodities. Innov Food Sci Emerg Technol 2008; 9:949-56.

29. Centeno S, Calvo MA, Adelantado C, Figueroa S. Antifungal activity of extracts of Rosmarium officinalis and Thymus vulgaris against Aspergillus flavus and A. ochraceus. Pak J Biol Sci 2010; 13:452-5.

30. Adam K, Sivropoulu A, Kokkini S, Lanaras T, Arsenakis M. Antifungal activities of Origanum vulgarae subsp. Hirtum, Mentha spicata, Lavandula angustifolia and Salvia fruticosa essential oils against human pathogenic fungi. $J$ Agric Food Chem 1998; 46:1739-45. 